\title{
Experimental study on seismic response of subway station built in loess
}

\section{Dengzhou Quan $^{\mathrm{a}}$ (i) Shaobo Chai ${ }^{\mathrm{a}^{*}}$ (D), Su Chen ${ }^{\mathrm{b}}$ (D), Yihong Wang}

a School of Civil Engineering, Chang' an University, Xi'an, 710064, China. E-mail: qdz0809@chd.edu.cn, shbchai@chd.edu.cn, wangyh@chd.edu.cn

b Institute of Geophysics, China Earthquake Administration, Beijing, 100081, China. E-mail: chensuchina@126.com

*Corresponding author

http://dx.doi.org/10.1590/1679-78256042

\begin{abstract}
The present investigation is concerned with the dynamic seismic response of subway station built in loess site by a series of shaking table tests. Firstly, according to the Bockingham $\pi$ theorem, the scale ratio is determined and then the model system is designed. Then, based on the geological environment and seismological background of Xi'an, the input ground motions and loading scheme are determined. On the basis of the test data, the acceleration responses of model system, strain response characteristics of the structure, distribution of dynamic soil pressure between loess and structure, the settlement of model ground and the seismic damage mode of model system are analyzed systematically. The results show that the peak accelerations in model soil increase gradually from the bottom to the top of the soil. The peak tensile strains measured at the top and bottom of the center columns are larger than those obtained at the side walls, while the peak tensile strains in the floor slab are the smallest. Moreover, the relationship between structure uplift and soil pressure difference can be fitted by exponential function.
\end{abstract}

Keywords

Shaking table test; Soil-structure interaction; Subway station; Loess area; Seismic response

\section{Graphical Abstract}
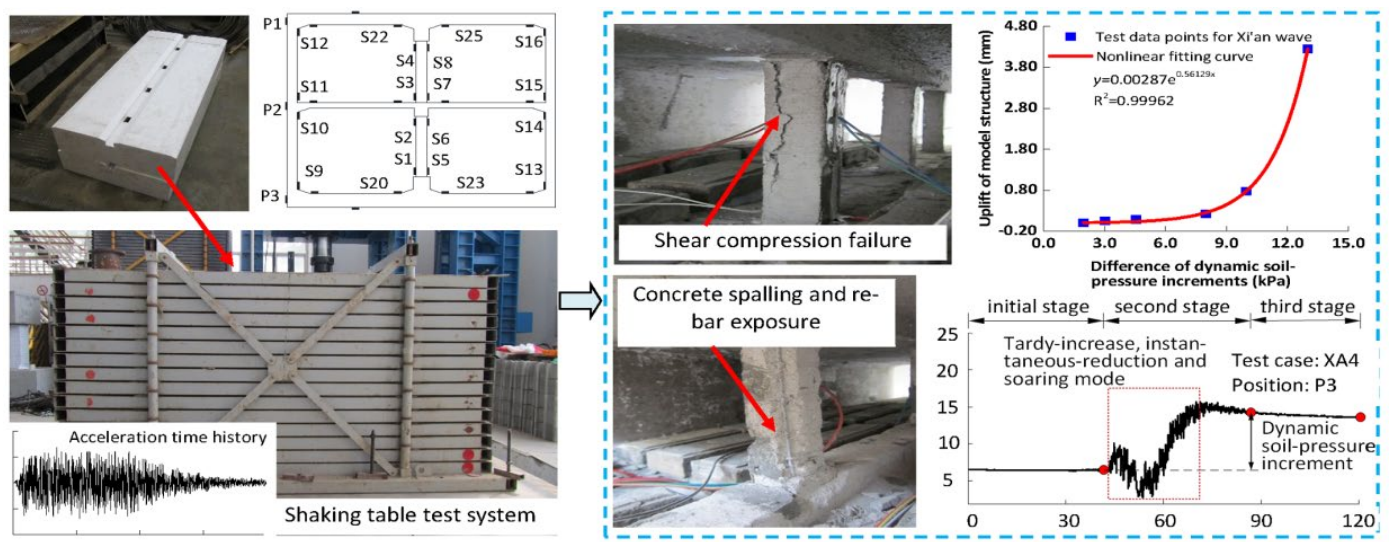


\section{INTRODUCTION}

With the rapid development of urbanization in China, the number of subway projects in loess area, such as Xi'an, Lanzhou, Taiyuan et al (Quan et al., 2016), is increasing gradually. Thus, the seismic response and safety evaluation of underground subway structures in loess areas has become a major concern (Quan et al., 2015). Generally, earthquake disaster is more serious in loess area than in other types of soil condition due to the special characteristics of loess such as columnar joint structure, macropores, weak cementation and the special sensitivity to water (Wang et al., 2010). The seismic subsidence, compaction and collapsible deformation of loess are induced by dynamic action, static force and soaking process respectively, and the magnitude of seismic subsidence has a close relation to the infliction sequence between dynamic action and other processes. Therefore, the dynamic behavior of loess is more sensitive to earthquake action. Accordingly, it is of great significance to investigate seismic response of underground subway structures built in loess site by carrying out shaking table tests.

In the last few years, seismic response characteristics of underground structures have been deeply and extensively studied by shaking and centrifuge table tests to check current design and analysis methods (Such as: Cilingir and Madabhushi, 2011; Varghese and Madhavi Latha, 2014; Baziar et al., 2014; Dashti et al., 2016; Rabeti Moghadam and Baziar, 2016; Farahi et al., 2018; Ritesh et al., 2019). Tamari and Towhata (2003) launched a series of shaking table tests on a flexible rectangular cross-section structure in liquefiable ground. The results showed that the soil-structure interaction of model system was significantly influenced by natural vibration period of ground and swelling characteristics of backfill. Ma et al. (2017) conducted shaking table tests on subway station joint structure to investigate the seismic response of the joint structure in soft soil. Jafarzadeh et al. (2010) carried out shaking table tests on buried pipeline in liquefiable ground, and found that the internal force of buried pipeline was significantly affected by the peak acceleration of input ground motion and soil conditions. Several researchers have conducted centrifuge shaking table tests on one-story and three-span subway stations, circular tunnels and one-story and two-span subway stations respectively, and the disaster mechanism of underground subway structures during earthquake was revealed by the series model tests (Han, 2011; Ling et al., 2012; Wang et al., 2013). Tsinidis et al. (2013) analyzed the seismic response of square section tunnel in sandy soil by centrifuge shaking table model tests, which indicated the swaying movement pattern of the tunnel in earthquake. In addition, some researches have been conducted to investigate the upward movement of model structure buried in liquefiable sand soil by centrifuge shaking table tests (e.g., Koseki et al., 1997; Sasaki et al., 1999; Adalier et al., 2003; Tobita et al., 2011; Chian and Madabhushi, 2012; Lanzano et al., 2012; Kang et al., 2014). Chen et al. $(2007,2013,2015)$ conducted a series of large-scale shaking table tests on underground subway structures with various cross sections in liquefiable ground. Based on the substantial amount of data recorded during tests including acceleration of model system, excess pore pressure, ground settlement, strains of the model structure and dynamic soil pressure, the seismic response and failure characteristics of underground subway structures were analyzed systematically. Liu et al. $(2017,2018)$ carried out series of shaking table tests to reveal the seismic response of an active ground fissure and failure mechanism of subway tunnel crossing the fissure. Hamayoon et al. (2018) studied the effect of partial ground improvement (PGI) as a seismic countermeasure for the existing box culverts and proposed the optimum pattern of PGI. Zhuang et al. (2019) conducted shaking table tests to study the seismic response of the connecting part between a subway station and a running tunnel located under a slightly inclined liquefiable ground. The results show apparent asymmetric liquefaction distribution characteristics in the surrounding soil on both sides of the subway station. These researches revealed the seismic performance of underground structures under different soil conditions, provided a reference to some extent for the design and construction of underground structures under the corresponding geological conditions.

However, these studies mentioned above only focused on the behaviors of underground structures located in sandy soil, soft soil or other liquefiable soil. Few experimental results or numerical simulations (e.g., Qin, 2010; Liu et al., 2017 \& 2018) are available in the literature on seismic performance of underground structures located in loess site. In this research, a series of shaking table tests on a scaled model of subway stations in loess site are designed and conducted. The main purpose of the series of shaking table model tests is to better understand the seismic response characteristics of underground structures in loess, such as the acceleration response of structures, the strain distribution of structures, the vertical settlement of loess site, and the dynamic soil pressures between loess and structure. The results can provide scientific basis for the seismic design of subway station in loess areas in some extent. 


\section{SHAKING TABLE TEST}

\subsection{Test apparatus and similitude ratio design}

The shaking table tests of subway station in loess are conducted in Nanjing University of Technology by the high-performance shaking table, manufactured by MTS Corp. USA. In the tests, the laminar shear model box is used for simulating shear deformation of loess. The shaking table, whose dimensions are $3.36 \mathrm{~m} \times 4.86 \mathrm{~m}$ in plane, can input ground motions with different frequency from 0.1 to $50 \mathrm{~Hz}$. The maximum acceleration of shaking table is $1.0 \mathrm{~g}$ with a maximum bearing capacity of 25 tons, where $g$ is the gravitational acceleration. The net size of the laminar shear model box is $3.5 \mathrm{~m} \times 2.0 \mathrm{~m} \times 1.7 \mathrm{~m}$. By the design of horizontal free-slip boundary condition, the reflection and scattering of the seismic wave at the boundary of the box can be eliminated effectively during shaking table tests (Chen et al., 2013).

Considering soil-structure interaction in the shaking table tests, the similarity relationship of subway station and the surrounding soil should be uniform as much as possible. Due to the limited space of the structure model, it is difficult to add full artificial weight to eliminate the gravity distortion effect of the model. As a result, the underweight artificial mass model is used in the model tests and the dynamical test model is designed according to the similitude laws recommended by Lin et al. (2000). Based on the characteristics of model structure and soils, the length, elastic modulus and acceleration are selected as basic physical quantities. The scale ratios of these basic physical quantities are determined according to the dimensions of the shaking table and dynamic performance of excitation system. The other physical quantities of the model structure as well as the model soil, such as the mass, density and wave velocity, can be deduced from Bockingham $\pi$ theorem (Li et al., 1996; Meymand, 1998). The similarity ratios of model system are shown in Table 1.

Table 1 Similarity ratios of the model system

\begin{tabular}{|c|c|c|c|c|}
\hline \multirow{2}{*}{ Types } & \multirow{2}{*}{ Physical quantity } & \multirow{2}{*}{ Similitude relation } & \multicolumn{2}{|c|}{ Similitude ratio } \\
\hline & & & Model structure & Model soil \\
\hline \multirow[t]{3}{*}{ Geometry properties } & Length I & $S_{1}$ & $1 / 30$ & $1 / 4$ \\
\hline & Area $A$ & $S_{A}=S_{1}^{2}$ & $1 / 900$ & $1 / 16$ \\
\hline & Linear displacement $r$ & $S_{r}=S_{l}$ & $1 / 30$ & $1 / 4$ \\
\hline \multirow[t]{4}{*}{ Material properties } & Elastic modulus $E$ & $S_{E}$ & $1 / 5$ & - \\
\hline & Stress $\sigma$ & $S_{\sigma}=S_{E}$ & $1 / 5$ & - \\
\hline & Equivalent density $\rho$ & $S_{\rho}=S_{E} /\left(S_{I} S_{a}\right)$ & 3 & 1 \\
\hline & Mass $m$ & $S_{m}=S_{\sigma} S_{1}^{2} / S_{a}$ & $1.11 \times 10-4$ & - \\
\hline \multirow[t]{3}{*}{ Mechanical Properties } & Concentrated force $F$ & $S_{F}=S_{\sigma} S_{T}^{2}$ & $2.22 \times 10-4$ & - \\
\hline & Linear load $q$ & $S_{q}=S_{\sigma} S_{I}$ & $6.67 \times 10-3$ & - \\
\hline & Moment $M$ & $S_{M}=S_{\sigma} S_{1}^{3}$ & $7.41 \times 10-6$ & - \\
\hline \multirow[t]{4}{*}{ Dynamic properties } & Acceleration $a$ & $S_{a}$ & 2 & 2 \\
\hline & Duration $t$ & $S_{t}=S_{I}^{0.5} S_{a}^{-0.5}$ & 0.1291 & 0.3536 \\
\hline & Frequency $\omega$ & $S_{\omega}=1 / S_{t}$ & 7.7460 & 2.8284 \\
\hline & Velocity $v$ & $S_{V}=S_{I}^{0.5} S_{a}^{0.5}$ & 0.2282 & 0.7071 \\
\hline
\end{tabular}

\subsection{The design of the model structure and the model soil}

The prototype structure of the shaking table tests is Feitian Road Station of Xi'an Metro Line 4, which is built in the loess tableland and designed as typical section of two-story and two-span. The micro-concrete and the galvanized steel wire are used to simulate the concrete and steel rebar of the prototype structure, respectively. Before the shaking table tests, the mix proportion and compressive yield strength of micro-concrete have been obtained through compression tests on the micro-concrete specimen with a size of $70.7 \mathrm{~mm} \times 70.7 \mathrm{~mm} \times 70.7 \mathrm{~mm}$. In addition, the elastic modulus of micro concrete specimen with the size of $70.7 \mathrm{~mm} \times 70.7 \mathrm{~mm} \times 210 \mathrm{~mm}$ has been tested, and the elastic modulus of micro concrete has been obtained. The tensile yield strength of micro-concrete, estimated by the code for designing concrete in China (GB50010-2010), is generally equal to $1 / 10^{\text {th }}$ compressive yield strength. The mix proportion and mechanical properties of the micro-concrete are shown in Table 2. The tensile yield strength and elasticity modulus of galvanized steel are approximately $1.19 \mathrm{GPa}$ and $200 \mathrm{GPa}$, respectively. In order to satisfy the similarity ratios of model structure, the rectangle lead blocks with the three-dimensional size of $7 \mathrm{~cm} \times 14 \mathrm{~cm} \times 3 \mathrm{~cm}$ are uniformly placed on the model structure. Because the total additional mass of the lead blocks is $465 \mathrm{~kg}$, i.e., $41.5 \%$ of the full artificial mass, it can realize 3 times equivalent density. To avoid the surrounding soil falling into the model structure, the ends of model structure were sealed with Plexiglas plate of $10 \mathrm{~mm}$ thickness. The manufacturing process of model structure is shown in Fig. 1. 
Table 2 Mix proportion and mechanical properties of the micro-concrete

\begin{tabular}{cccc}
\hline Mix proportion & $\begin{array}{c}\text { Compressive yield } \\
\text { strength (MPa) }\end{array}$ & $\begin{array}{c}\text { Tensile yield } \\
\text { strength (MPa) }\end{array}$ & $\begin{array}{c}\text { Elastic modulus } \\
(\mathrm{GPa})\end{array}$ \\
\hline $\begin{array}{c}\text { Cement: fine aggregate: coarse aggregate: hydrated lime: } \\
\text { water=1:6.5:0.5:0.5:1.4 }\end{array}$ & 6.1 & 0.61 & 6.6 \\
\hline
\end{tabular}

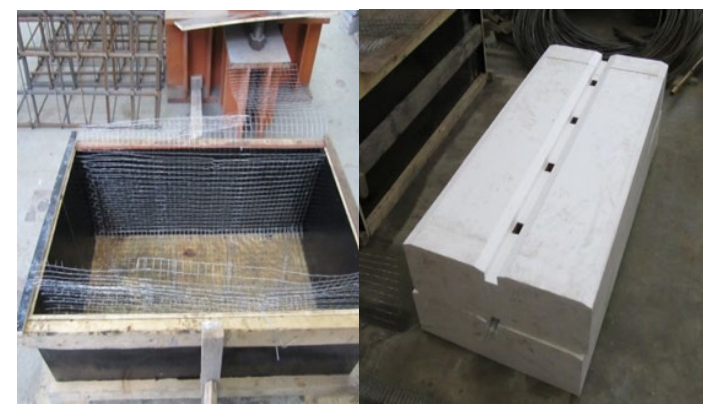

Inner and exterior formword

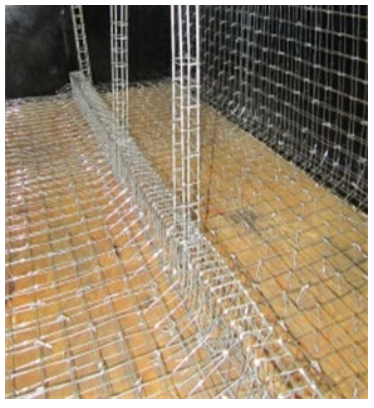

Assembling reinforcement

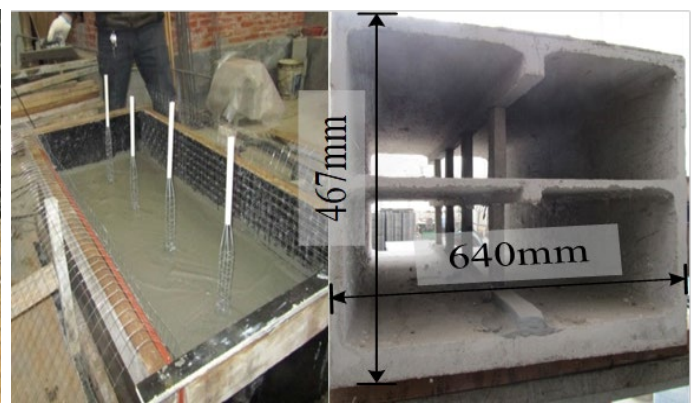

Concreting and concrete curing

Figure 1 Manufacturing process of model structure

In order to simulate the dynamic characteristics of loess sites, the model soil is collected from foundation pit excavation of Feitian Road Station (Xi'an Metro Line 4). After some simple treatment, the soil is placed in the laminar shear model box layer by layer. In order to avoid the model soil becoming too dry due to water loss and ensure that the water content of the model soil is basically the same as the natural water content of the original site, the model soil needs to be humidified. According to the difference between the water content of model soil and the natural water content, as well as the measured total mass and water content of model soil, the total amount of water needed to be added can be estimated. The water is added to the model soil in several times by a watering can. After each quick mixing with water, the model soil was covered with plastic cloth immediately, and the side of the filling box was sealed. Then, it is left standing for 72 hours, and the water content of the model soil need to be tested after the water in the model soil is fully diffused and uniform. In this way, when the soil sample water content is slightly higher than the natural water content of the prototype site, water addition will be stopped, considering the moisture loss in the process of model soil packing.

In the process of making model soil, the model soil is layered into the model box, and wooden boards are placed on the top of each newly filled loose soil layer for human power trampling and compaction. In order to ensure that the density of the model soil is the same as the natural density of the original site, the thickness of each newly filled layer of loose soil should not be greater than $200 \mathrm{~mm}$. The expected thickness of soil layer after compaction can be calculated according to the weight of the filled soil and the natural density of soil in the original site, which is used as the compaction control index. The compaction effect is controlled by testing the thickness and density of soil layer. At the same time, in order to ensure a good bonding performance between adjacent soil layers, the top surface of each layer of model soil is roughed after compaction. After preparation, the model soil has been standing for 7 days in its natural state in the laboratory. The properties of the loess used in the test are measured by conventional soil tests, shown in Table 3. The manufacturing process of model soil is shown in Fig. 2.

Table 3 Physical parameters of soil samples

\begin{tabular}{ccccccccc}
\hline $\begin{array}{c}\text { Unit weight } \\
\left(\mathbf{k N} / \mathbf{m}^{\mathbf{3}}\right)\end{array}$ & $\begin{array}{c}\text { Dry density } \\
\left(\mathbf{g} / \mathbf{c m}^{3}\right)\end{array}$ & $\begin{array}{c}\text { Water content } \\
(\mathbf{\%})\end{array}$ & $\begin{array}{c}\text { Initial } \\
\text { void ratio }\end{array}$ & $\begin{array}{c}\text { Liquid limit } \\
(\%)\end{array}$ & $\begin{array}{c}\text { Plastic limit } \\
(\%)\end{array}$ & $\begin{array}{c}\text { Plasticity } \\
\text { index }\end{array}$ & $\begin{array}{c}\text { Cohesion } \\
(\mathbf{k P a})\end{array}$ & $\begin{array}{c}\text { Friction angle } \\
(\boldsymbol{*})\end{array}$ \\
\hline 16.7 & 1.41 & 22.7 & 0.973 & 34.2 & 20.3 & 13.9 & 29.0 & 21.0 \\
\hline
\end{tabular}




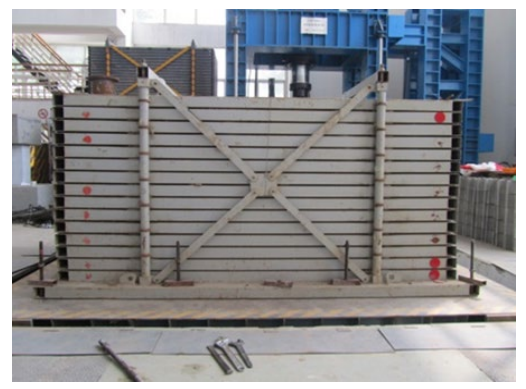

Laminar shear model box

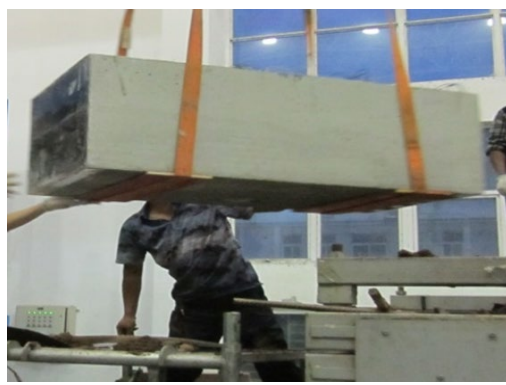

Loading soil and hoisting structure

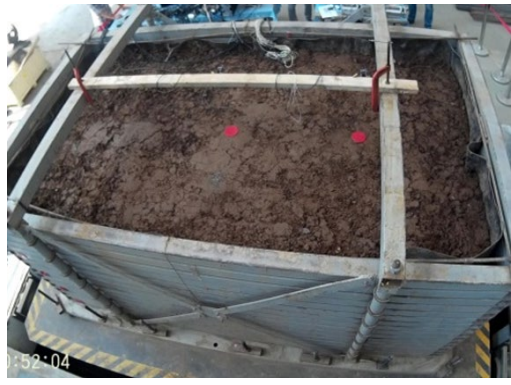

Completion of model soil

Figure 2 Manufacturing process of model soil

\subsection{Sensor layout}

In this present research, dynamic response characteristics of the model structure and soil are studied by recording and analyzing various parameters during the series of shaking table tests, such as the accelerations, the horizontal displacements and the vertical settlements of model system. Besides, the strain response of model structure and the dynamic soil pressure between loess and structure are also investigated. Before the tests, the seismic responses of subway station built in loess have been investigated by finite element numerical simulation (Quan et al., 2015). Accordingly, the seismic response characteristics of model system are preliminary revealed and the reasonable sensors placement schemes of the tests are determined to obtain reliable data during the shaking table tests of subway station in loess. The positions of observation sections and sensor layout of the shaking table tests are shown in Fig. 3 . There are 6 observation sections arranged in the model structure, one of them was selected as the primary observation section and the others served as secondary observation section.

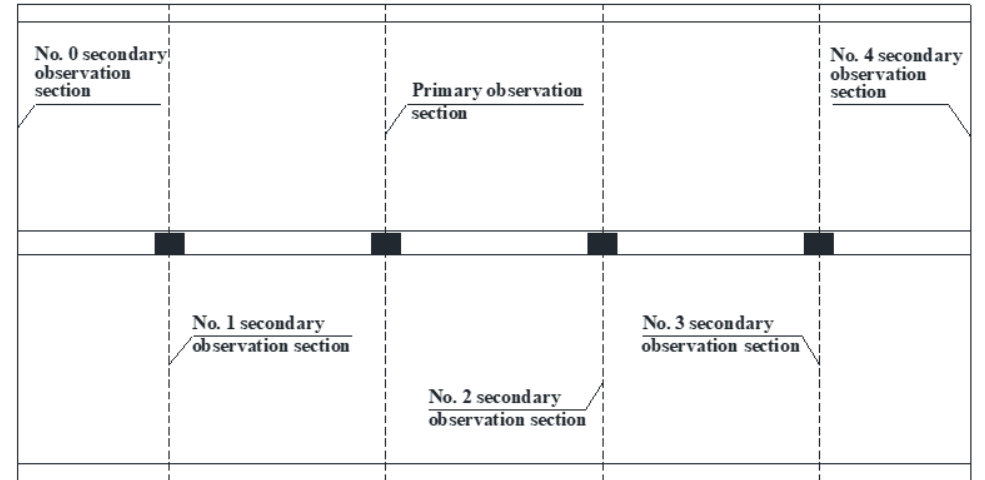

(a) Location of observation sections in model structure

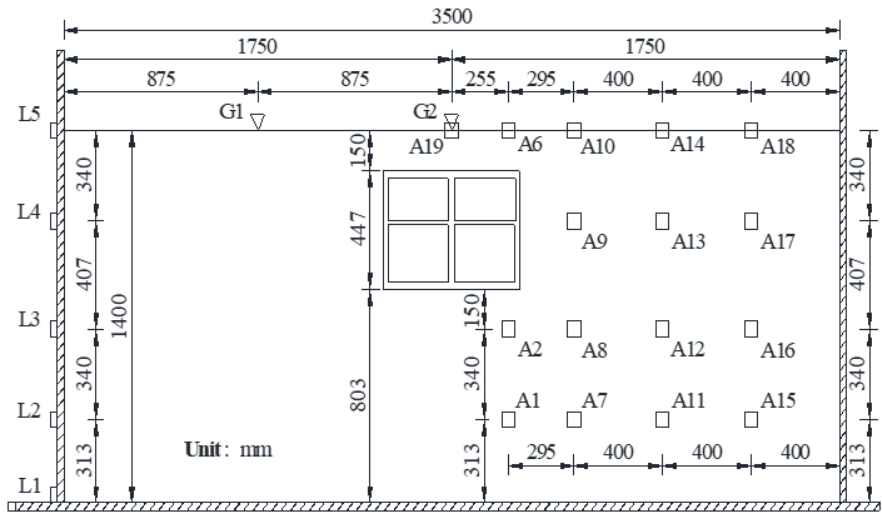

(c) Sensor of model soil in primary observation section

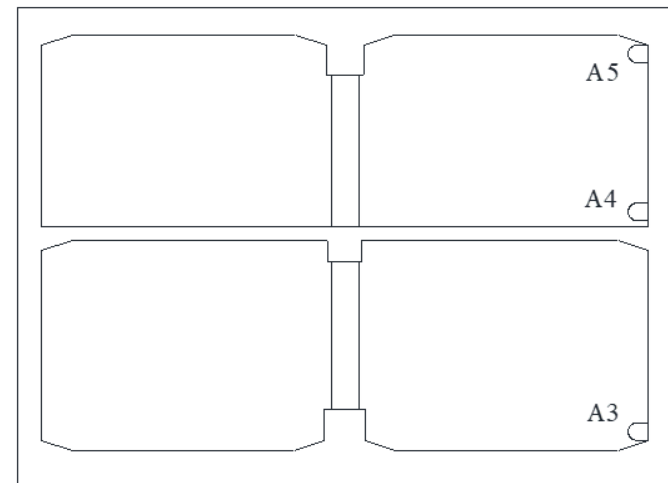

(b) Accelerometer in primary observation section

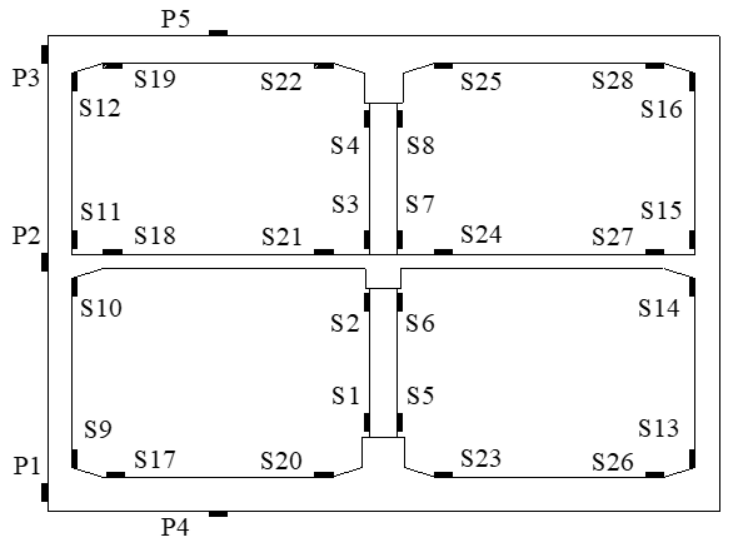

(d) Strain gauges in primary observation section

Figure 3: Observation sections and sensor layout of the shaking table tests. 


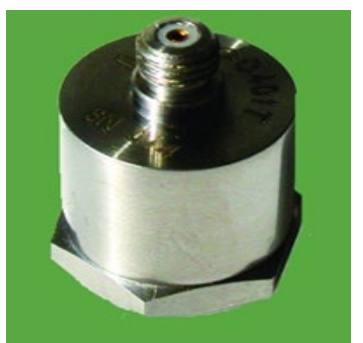

Accelerometer fixed in model structure

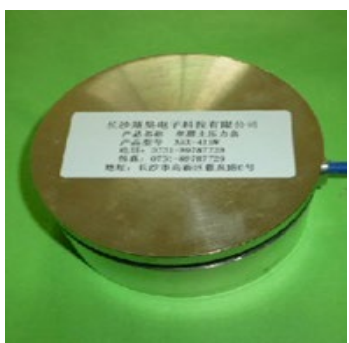

Soil pressure transducers

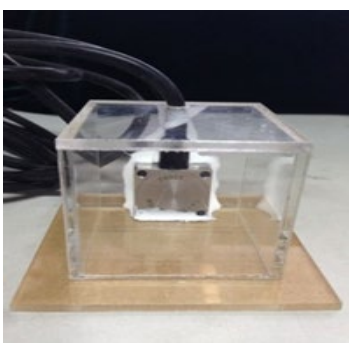

Accelerometer fixed in model soil

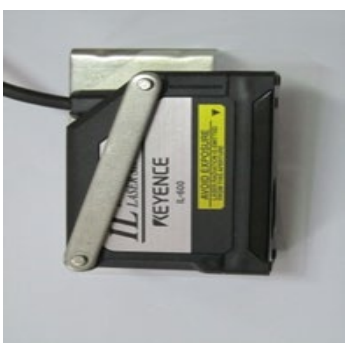

Laser sensors

Figure 4 Sensors used in the shaking table test

The points $A n(n=[1,26])$ in Fig. 3(b) and Fig. 3(c) represent accelerometers, which are located in 26 test points. The points A3, A4 and A5 are fixed in primary observation section of model structure. The points A20, A21 and A22 are fixed in No. 2 secondary observation section with the same layout as A3, A4 and A5 in primary observation section. The point A23 is placed in No. 3 secondary observation section with the same location as A5 in primary observation section. The rest of accelerometers are embedded in the model soil, as shown in Fig.3(c). The other types of sensors fixed in model system are also shown in Fig. 3(c) and Fig. 3(d), which includes forty-four strain gauges, seven soil pressure transducers, five displacement transducers and two laser sensors (used for settlement measuring), denoted as S, P, $L$ and $G$, respectively. Sensors used in the shaking table tests are shown in Fig. 4.

\subsection{Input ground motions and test scheme}

To explore the seismic performance of underground subway station in loess, seismic records with different frequency spectrum characteristics and durations are selected as input ground motions in the series of shaking table tests. The original ground motions used in the tests are Taft ground motion, Songpan ground motion and Xi' an artificial wave, and the acceleration time histories and Fourier spectra of them are shown in Fig. 5.
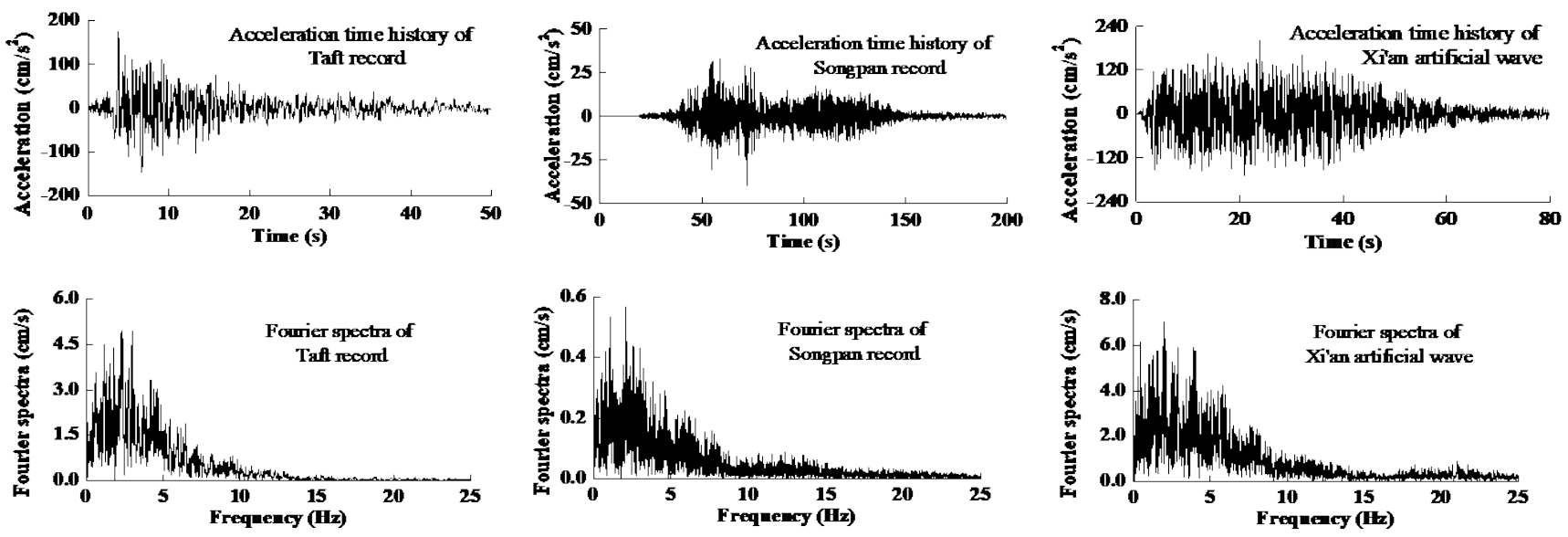

Figure 5 Time-histories and Fourier spectra of input ground motions

The peak acceleration, fault distance and duration of original Taft ground motion, recorded the Taft seismologic recording station (No. 1095) during the Ms7.7 Kern County earthquake on 21 July 1952 in California, USA, are $175.9 \mathrm{~cm} / \mathrm{s}^{2}, 43.5 \mathrm{~km}$ and $54 \mathrm{~s}$, respectively. The corresponding parameters of original Songpan ground motion, recorded at 51SPT seismologic recording station during the Ms 8.0 WenChuan earthquake on 12 May 2008 in Sichuan Province, China, are $40.2 \mathrm{~cm} / \mathrm{s}^{2}, 122 \mathrm{~km}$ and 213s, respectively. Xi' an artificial wave is synthesized according to characteristic of the loess site with probability analysis method. The peak acceleration and duration of artificial wave are $200.6 \mathrm{~cm} / \mathrm{s}^{2}$ and $60 \mathrm{~s}$, respectively. Based on the original seismic records and artificial wave, the peak ground accelerations (PGA) of input motions are adjusted to $0.05 \mathrm{~g}, 0.1 \mathrm{~g}, 0.2 \mathrm{~g}, 0.4 \mathrm{~g}, 0.6 \mathrm{~g}, 0.8 \mathrm{~g}$ and $1.2 \mathrm{~g}$ gradually during the shaking table tests. The test schemes are shown in Table 4. 
Table 4 Loading conditions for shaking table test

\begin{tabular}{ccccccccc}
\hline Test No. & Input motions & PGA (g) & Test No. & Input motions & PGA (g) & Test No. & Input motions & PGA (g) \\
\hline B0 & Flat noise & 0.05 & SP3 & Songpan record & 0.2 & TF5 & Taft record & 0.6 \\
SP1 & Songpan record & 0.05 & TF3 & Taft record & 0.2 & XA5 & Artificial wave & 0.6 \\
TF1 & Taft record & 0.05 & XA3 & Artificial wave & 0.2 & B5 & Flat noise & 0.05 \\
XA1 & Artificial wave & 0.05 & B3 & Flat noise & 0.05 & SP6 & Songpan record & 0.8 \\
B1 & Flat noise & 0.05 & SP4 & Songpan record & 0.4 & TF6 & Taft record & 0.8 \\
SP2 & Songpan record & 0.1 & TF4 & Taft record & 0.4 & XA6 & Artificial wave & 0.8 \\
TF2 & Taft record & 0.1 & XA4 & Artificial wave & 0.4 & B6 & Flat noise & 0.05 \\
XA2 & Artificial wave & 0.1 & B4 & Flat noise & 0.05 & XA7 & Artificial wave & 1.2 \\
B2 & Flat noise & 0.05 & SP5 & Songpan record & 0.6 & B7 & Flat noise & 0.05 \\
\hline
\end{tabular}

\section{RESULTS AND INTERPRETATION}

\subsection{Acceleration response of model system}

\section{(1) Acceleration response of model soil}

From the accelerometers A11, A12, A13 and A14, which are embedded uniformly in the model soil (as shown in Fig. $\left.3^{\odot}\right)$, abundant data can be obtained to investigate the seismic response characteristics of loess site during the tests. Fig. 6 presents the peak accelerations of model soil in different test cases. It can be observed that the peak accelerations of model soil at same depth increases with the PGA of input motions increase. Mostly, the peak accelerations induced by the same PGA of input motions increases from the bottom to the top of model soil, which illustrated the amplification effect of the soil (Fig. 6(a)). For the cases with $P G A=0.1 \mathrm{~g}$, the peak accelerations of model soil are not significantly different under input motions with different frequency spectrum characteristics and all of them lay between $0.08 \mathrm{~g}$ and 0.22 g (Fig. 6(b)).

However, for the test cases with a higher PGA (e.g., PGA=0.4g, $0.6 \mathrm{~g}$ or $0.8 \mathrm{~g}$ ), the peak accelerations of model soil with different depth vary totally under different input motions. As shown in Fig. $6^{\odot}$, the peak accelerations induced by the Xi'an artificial waves are greater than those induced by the Songpan and Taft ground motions at a same depth. The results indicate that the seismic response of model soil is significantly affected by the frequency spectrum characteristics of input waves under strong ground motions. For the same PGA, the seismic response of the model soil is more sensitive to strong ground motions with larger low frequency components.

Peak acceleration (g)

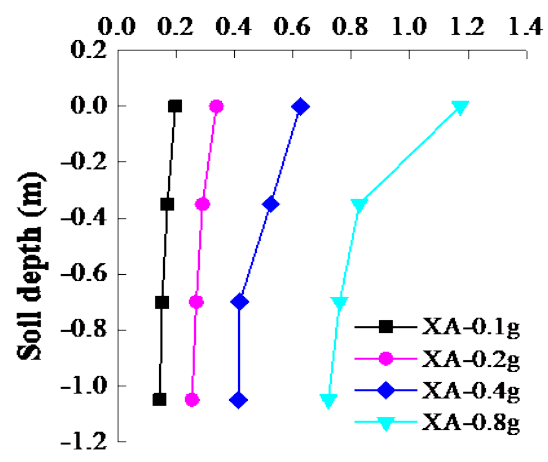

(a) Results during Xi'an wave
Peak acceleration (g)

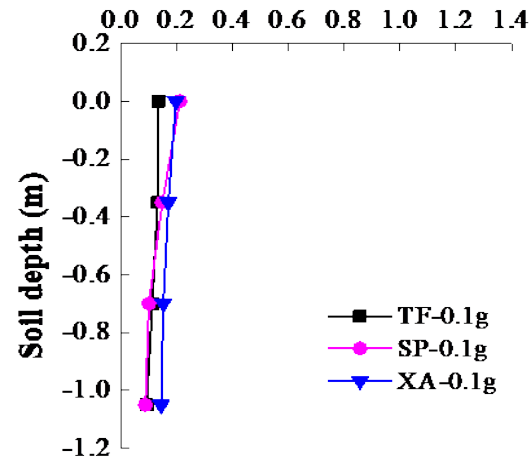

(b) Results for PGA $=0.1 \mathrm{~g}$
Peak acceleration (g)

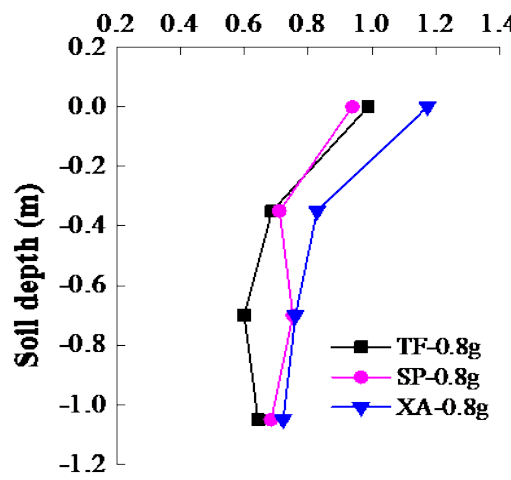

(c) Results for PGA $=0.8 \mathrm{~g}$

Figure 6: Peak accelerations of model soil under different ground motions 


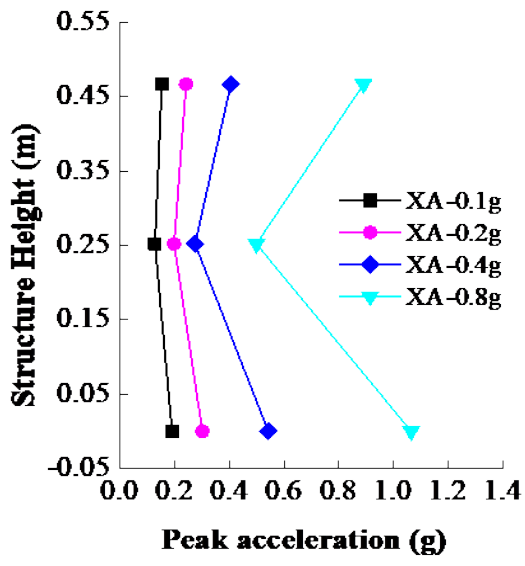

(a) Results during Xi'an wave

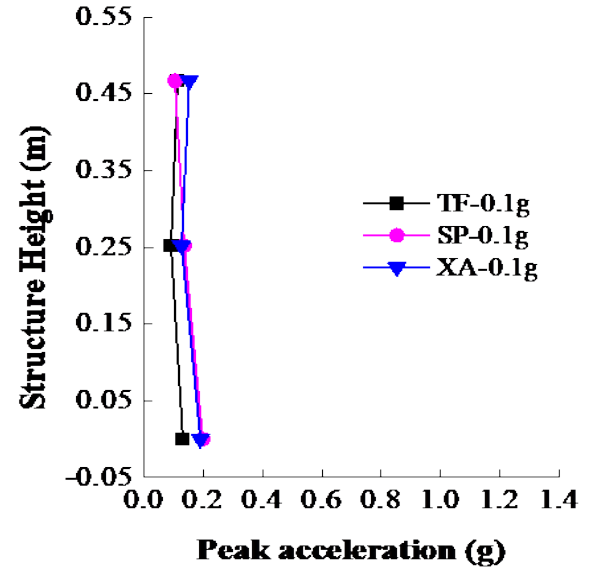

(b) Results for $\mathrm{PGA}=0.1 \mathrm{~g}$

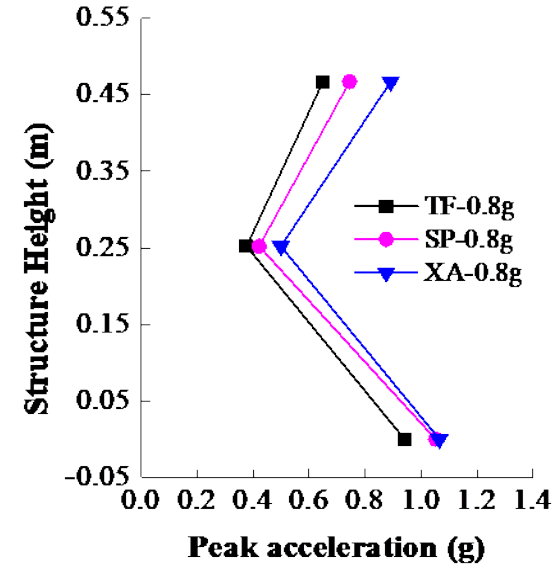

(c) Results for $\mathrm{PGA}=0.8 \mathrm{~g}$

Figure 7: Peak accelerations of model structure under different ground motions

\section{(2) Acceleration response of model structures}

The seismic response characteristics of model structure are investigated by analyzing the data recorded by the accelerometers A20, A21 and A22. As shown in Fig. 3, the three accelerometers are placed in the bottom, middle and top of the structure, respectively. Fig. 7 shows the results of peak acceleration in model structure with different test cases. From Fig. 7(a), the peak accelerations of model structure at the same height increased observably as the increase of PGA of input motions. When the PGA of input motion is less than or equal to $0.4 \mathrm{~g}$, the peak accelerations have little changes with the heights of the monitoring point in the structure. The main reason is that the soil-structure interaction is not prominent under a weak ground motion and the movement of model system is coordinating in the tests. However, under strong ground motions, the soil-structure interaction become intense, incongruous movement of model system appears, resulting in the occasional separation between model structure and the surrounding soil. When the PGA of input motions exceeds $0.4 \mathrm{~g}$, both the peak accelerations in the bottom and in the top of model structure are greater than that in the middle of the structure. In addition, the peak acceleration in the top of model structure is slightly less than that in the bottom of the structure. The reason is that the concentrated gravity in the middle of the structure is far less than that in other parts and the concentrated gravity in the top of the structure is slightly smaller than that in the bottom of the structure. Accordingly, it is possible to conclude that the gravity distribution of model structure has an important influence on its seismic response characteristics in the incongruous movement.

As shown in Fig. 7(b), PGA=0.1 g, the peak accelerations of model structure are not much different under input motions with different frequency spectrum characteristics. However, for test cases with a higher PGA, such as PGA=0.8 g, the peak accelerations of model structure with different height vary totally under different input ground motions, as shown in Fig. 7(c). The peak accelerations induced by the Xi'an artificial waves are greater than that induced by the Songpan and Taft ground motions. The response characteristics of peak accelerations in the model structure are similar to that in the model soil under input motions with different frequency spectrum, which indicates that the seismic response of the underground structure is significantly affected by the dynamic response of surrounding loess during the earthquake.

\subsection{Strain distribution of model structures}

Under the Songpan ground motions, the strain amplitudes of model structure in primary observation section are shown in Table 5. It is clear that the strain amplitudes of all the measuring points in the structure increase basically with the PGA of input motions rising. From the overall perspective, the strain amplitudes in top and bottom plate are smaller than those in side walls which are smaller than that in central column. In addition, the strain amplitudes in middle plate are larger than those in other plates due to the gravity and stiffness distribution of model structure. The lateral stiffness of central column and middle plate are relative small, which results in larger strain amplitudes during dynamic soil-structure interaction. 
Table 5 Strain amplitudes of model structure in primary observation section

\begin{tabular}{|c|c|c|c|c|c|c|c|c|c|c|c|c|}
\hline \multirow{2}{*}{\multicolumn{2}{|c|}{ Position }} & \multicolumn{2}{|c|}{ No: SP2 } & \multicolumn{2}{|c|}{ No: SP3 } & \multicolumn{2}{|c|}{ No: SP4 } & \multicolumn{2}{|c|}{ No: SP5 } & \multicolumn{2}{|c|}{ No: SP6 } & \multirow{2}{*}{ Schematic diagram } \\
\hline & & $\mathrm{C}^{*}$ & $\mathbf{T}$ & C & $T$ & C & $T$ & C & $T$ & C & $T$ & \\
\hline \multirow{4}{*}{$\begin{array}{l}\text { Central } \\
\text { column }\end{array}$} & S4 & -91 & 61 & -125 & 62 & -330 & 136 & -546 & 209 & -901 & 212 & \multirow{2}{*}{$\begin{array}{l}\mathrm{S} 4 \\
\mathrm{~S} 3\end{array}$} \\
\hline & S3 & -19 & 19 & -38 & 27 & -175 & 111 & -492 & 364 & -965 & 709 & \\
\hline & S2 & -17 & 17 & -29 & 26 & -127 & 33 & -70 & 29 & -194 & 130 & \multirow{2}{*}{$\begin{array}{l}\text { S2 } \\
\text { S1 }\end{array}$} \\
\hline & S1 & -80 & 48 & -86 & 58 & -143 & 103 & -201 & 125 & -223 & 77 & \\
\hline \multirow[t]{4}{*}{ Side wall } & S16 & -73 & 62 & -105 & 84 & -359 & 326 & -458 & 502 & -425 & 315 & S16 \\
\hline & $\mathrm{S} 15$ & -35 & 27 & -26 & 34 & -44 & 70 & -47 & 4 & -33 & 44 & $\mathrm{~S} 15$ \\
\hline & S14 & -28 & 11 & -26 & 0 & -18 & 26 & -33 & 18 & -66 & 15 & S14 \\
\hline & S13 & -46 & 43 & -100 & 76 & -244 & 122 & -440 & 413 & -710 & 578 & \\
\hline \multirow[t]{3}{*}{ Top plate } & S19 & -13 & 13 & -24 & 23 & -51 & 120 & -50 & 143 & -62 & 37 & \multirow{2}{*}{\begin{tabular}{|ll|ll|}
$\overline{\mathrm{S} 19}$ & $\overline{\mathrm{S} 22}$ & $\overline{\mathrm{S} 25}$ & $\overline{\mathrm{S} 28}$ \\
& & (lost) \\
\end{tabular}} \\
\hline & $\mathrm{S} 22$ & -10 & 12 & -22 & 13 & -18 & 23 & -58 & 39 & -75 & 21 & \\
\hline & $\mathrm{S} 25$ & -11 & 10 & -18 & 22 & -18 & 73 & -18 & 33 & -55 & 18 & \\
\hline \multirow{4}{*}{$\begin{array}{l}\text { Middle } \\
\text { plate }\end{array}$} & $\mathrm{S} 18$ & -63 & 88 & -93 & 112 & -266 & 421 & -251 & 1213 & -384 & 1618 & \multirow{4}{*}{\begin{tabular}{ll|ll|} 
S18 & S21 & S24 & S27 \\
\end{tabular}} \\
\hline & $\mathrm{S} 21$ & -25 & 13 & -40 & 15 & -187 & 179 & -315 & 256 & -410 & 304 & \\
\hline & $\mathrm{S} 24$ & -24 & 20 & -29 & 26 & -449 & 304 & -773 & 370 & -971 & 238 & \\
\hline & S27 & -89 & 82 & -102 & 109 & -542 & 530 & -466 & 965 & -425 & 2146 & \\
\hline \multirow{4}{*}{$\begin{array}{l}\text { Bottom } \\
\text { plate }\end{array}$} & S17 & -13 & 13 & -14 & 19 & -51 & 37 & -62 & 26 & -70 & 7 & \\
\hline & $\mathrm{S} 20$ & -8 & 10 & -5 & 12 & -7 & 32 & -6 & 45 & -20 & 67 & \\
\hline & $\mathrm{S} 23$ & -10 & 7 & -4 & 11 & -29 & 4 & -48 & 0 & -88 & 0 & \multirow[b]{2}{*}{$\begin{array}{lllll}\mathrm{S} 17 & \mathrm{~S} 20 & \mathrm{~S} 23 & \mathrm{~S} 26 \\
\end{array}$} \\
\hline & $\mathrm{S} 26$ & -14 & 8 & -15 & 17 & -31 & 34 & -24 & 45 & -43 & 72 & \\
\hline
\end{tabular}

${ }^{*} \mathrm{C}$ means compressive strain; $\mathrm{T}$ means tension strain; The unit is $\mu \varepsilon$.

When the PGA of input motion is less than $0.4 \mathrm{~g}$, the model structure mainly exhibits elastic deformation characteristics. Due to the higher stiffness and the larger bending moment induced in the top plate, the strain amplitudes on the top of central column (S4) are larger than those on the bottom of central column (S3) in the upper storey of model structure. On the contrary, the strain amplitudes on the top of central column (S2) are smaller than those on the bottom of central column (S1) in the lower storey of model structure owing to the lesser stiffness and lower bending moment induced in the middle plate. During the tests, the dynamic soil-structure interaction in the upper storey is stronger, which results in the greater shear deformation between the top and middle plate. Accordingly, the strain amplitudes on the top of central pillars in the upper storey (S4) are larger than those on the bottom of central column in the lower storey (S1). From the results, it is observed that the strain amplitude of the joint between the side wall and the bottom plate (S13) is significantly larger than that of the joint between the side wall and the middle plate (S14 or S15)))), while slightly smaller than that of the joint between the side wall and the top plate (S16). This is because that the thickness of the middle plate in the subway station structure is relatively thinner, which means that the section stiffness is significantly smaller than that of the top plate and the bottom plate. As a result, the additional bending moment at the joint between the side wall and the middle plate is the smallest, which shows that the strain amplitude is the smallest. In addition, due to the large peak acceleration of the soil around the upper storey as well as the large shear deformation of the soil layer, the shear deformation of the lower storey (between the middle plate and the bottom plate) is smaller than that of the upper storey (between the top plate and the middle plate), resulting in the aboved result. Furthermore, the strain amplitudes in the plates nearby the side walls (S19, S18 or S17) are larger than those nearby the central columns (S22, S21 or S20) due to the higher stiffness and larger bending moment induced in side walls.

As the PGA of input motions increase, the dynamic soil-structure interaction becomes more intense gradually during the shaking table tests. When the PGA of input motions exceeds $0.4 \mathrm{~g}$, the dynamic damages of structural members are generated and the strain response of the structure has a great change. In the bottom plate, the compressive strain amplitudes of left end in the left span (S17) and left end in the right span (S23) increase dramatically and the tension strain amplitudes of right end in the left span (S20) and right end in the right span (S26) have an evident growth. It illustrates that the clockwise torsion and unrecoverable shear deformation occur in No. 2 secondary observation section of the structure during strong interacting between the model soil and structure. The similar phenomena can be observed in the top plate. From the working condition of SP5 (Songpan ground motions with PGA=0.6 g), the tensile damage of the junctions between the top plate and central column appears and the internal forces in the central column are redistributed. As a result, the bending moment and tension strain amplitudes on the top of central column (S4) are 
less than those on the bottom of central column (S3) in the upper storey. In the same way, from the case of SP6 (Songpan ground motions with PGA=0.8 g), the compressive strain amplitudes on the top of central column (S4) are less than those on the bottom of central column (S3) in the upper storey. For the lower storey, the tension strain amplitudes on the bottom of central column (S1) are less than those on the top of central column (S2). The tension strain amplitudes on the left end of top plate (S19) decrease observably. The reason for these above phenomena in the working condition of SP6 is because of the partial damage appearance in the model structure.

The strain amplitudes of model structure under input motions with different frequency spectrum are shown in Table 6 . The results indicate that the strain amplitudes induced by the Xi'an artificial waves are generally greater than those induced by the Songpan and Taft ground motions in all of strain test points. It can be concluded that the strain response of the model structure is more sensitive to input motions with larger low frequency components for the same PGA.

Table 6 Strain amplitudes of primary observation section under different input motions

\begin{tabular}{|c|c|c|c|c|c|c|c|c|c|c|c|c|c|c|}
\hline \multirow{2}{*}{\multicolumn{2}{|c|}{ Position }} & \multicolumn{2}{|c|}{ No: SP3 } & \multicolumn{2}{|c|}{ No: TF3 } & \multicolumn{2}{|c|}{ No: XA3 } & \multicolumn{2}{|c|}{ No: SP4 } & \multicolumn{2}{|c|}{ No: TF4 } & \multicolumn{2}{|c|}{ No: XA4 } & \multirow{2}{*}{ Schematic diagram } \\
\hline & & $\mathrm{C}^{*}$ & $\mathbf{T}$ & C & $\mathbf{T}$ & C & $\mathbf{T}$ & C & $\mathbf{T}$ & C & $\mathbf{T}$ & C & $\mathbf{T}$ & \\
\hline \multirow{4}{*}{$\begin{array}{l}\text { Central } \\
\text { column }\end{array}$} & S4 & -125 & 62 & -169 & 64 & -212 & 66 & -330 & 136 & -359 & 143 & -645 & 337 & \multirow{2}{*}{$\begin{array}{l}\mathrm{S} 4 \\
\mathrm{~S} 3 \mathrm{I}\end{array}$} \\
\hline & S3 & -38 & 27 & -52 & 44 & -56 & 44 & -175 & 111 & -201 & 139 & -476 & 331 & \\
\hline & S2 & -29 & 26 & -32 & 28 & -44 & 32 & -127 & 33 & -128 & 22 & -44 & 59 & \multirow{2}{*}{$\begin{array}{l}\mathrm{S} 2 \\
\mathrm{~S} 1\end{array}$} \\
\hline & S1 & -86 & 58 & -86 & 57 & -73 & 59 & -143 & 103 & -172 & 106 & -253 & 176 & \\
\hline \multirow{3}{*}{ Side wall } & S15 & -26 & 34 & -41 & 35 & -29 & 43 & -44 & 70 & -87 & 11 & -51 & 18 & $\mathrm{~S} 15$ \\
\hline & S14 & -26 & 0 & -36 & 14 & -11 & 11 & -18 & 26 & -29 & 26 & -22 & 18 & \\
\hline & S13 & -100 & 76 & -90 & 78 & -110 & 90 & -244 & 122 & -310 & 127 & -419 & 408 & I \\
\hline \multirow[t]{3}{*}{ Top plate } & S19 & -24 & 23 & -29 & 26 & -42 & 33 & -51 & 120 & -50 & 126 & -51 & 132 & \multirow{2}{*}{\begin{tabular}{|ll|rr|}
$\overline{\mathrm{S}} 19$ & $\mathrm{~S} 22$ & $\overline{\mathrm{S} 25}$ & $\begin{array}{r}\mathrm{S} 28 \\
\text { (lost) }\end{array}$ \\
\end{tabular}} \\
\hline & S22 & -22 & 13 & -25 & 13 & -40 & 20 & -18 & 23 & -44 & 27 & -26 & 34 & \\
\hline & S25 & -18 & 22 & -14 & 22 & -19 & 22 & -18 & 73 & -24 & 40 & -22 & 73 & \\
\hline \multirow{4}{*}{$\begin{array}{l}\text { Middle } \\
\text { plate }\end{array}$} & S18 & -93 & 112 & -95 & 116 & -158 & 126 & -266 & 421 & -126 & 949 & -69 & 1146 & \multirow{4}{*}{\begin{tabular}{ll|ll|}
$\mathrm{S} 18$ & S21 & S24 & S27 \\
\end{tabular}} \\
\hline & $\mathrm{S} 21$ & -40 & 15 & -55 & 27 & -59 & 66 & -187 & 179 & -201 & 190 & -407 & 355 & \\
\hline & S24 & -29 & 26 & -61 & 31 & -148 & 39 & -449 & 304 & -505 & 333 & -894 & 498 & \\
\hline & S27 & -102 & 109 & -92 & 111 & -341 & 114 & -542 & 530 & -598 & 679 & -617 & 935 & \\
\hline \multirow{4}{*}{$\begin{array}{l}\text { Bottom } \\
\text { plate }\end{array}$} & S17 & -14 & 19 & -14 & 15 & -37 & 23 & -51 & 37 & -99 & 42 & -66 & 51 & \\
\hline & S20 & -5 & 12 & -5 & 10 & -9 & 19 & -7 & 32 & -20 & 38 & -24 & 49 & \\
\hline & S23 & -4 & 11 & -11 & 12 & -18 & 10 & -29 & 4 & -29 & 4 & -51 & 0 & \multirow[b]{2}{*}{$\begin{array}{lllll}\mathrm{S} 17 & \mathrm{~S} 20 & \mathrm{~S} 23 & \mathrm{~S} 26 \\
\end{array}$} \\
\hline & S26 & -15 & 17 & -14 & 17 & -19 & 20 & -31 & 34 & -41 & 44 & -55 & 64 & \\
\hline
\end{tabular}

${ }^{*} \mathrm{C}$ means compressive strain; $\mathrm{T}$ means tension strain; The unit is $\mu \varepsilon$.

\subsection{Dynamic soil pressure between loess and structure}

The dynamic soil pressures between loess and structure are measured by the soil pressure transducers P1-P5. As shown in Fig. 3(d), the soil pressure transducers are placed in the primary observation section of model structure. Under the Xi'an artificial waves with the different PGA, the dynamic soil-pressure time-histories curves on the top of side wall (P3) are shown in Fig. 8, from which the development of dynamic soil pressures can be divided into three stages. During the first stage, the static pressure characteristics of soil pressures are observed and the value of dynamic soil pressures maintains a constant due to the coordinating movement between model soil and structure in this testing period. In the second stage, the accumulation and rapid increase of dynamic soil pressures occur due to the uncoordinated movement of model system such as relative sliding between model soil and structure. In the third stage, the dynamic soil pressures are keeping fixed values again which are equal to the values in the end of second stage. The reason is that the dynamic contact between model soil and structure show a stable state at the end of input ground motions. However, the tardy slow increase, instantaneous reduction and soaring are observed successively in the second stage of the dynamic soil pressures curve under the working condition of XA4. This phenomenon might be relevant to the intense soil-structure interaction and instantaneous separation between model structure and soil during strong ground motions. 


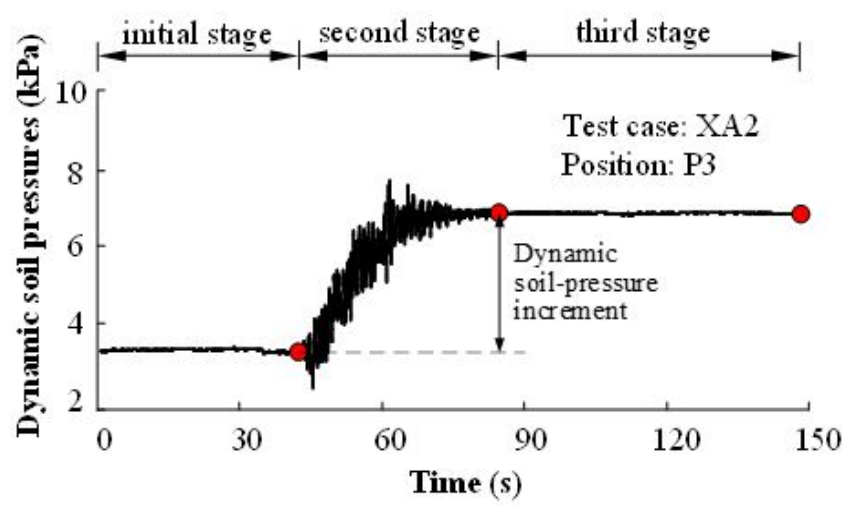

(a) Time-history curve during test case XA2

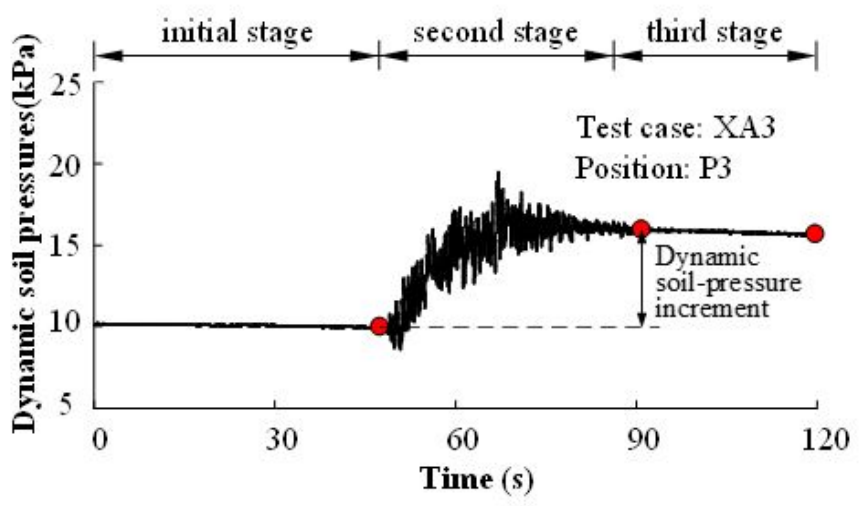

(b) Time-history curve during test case XA3

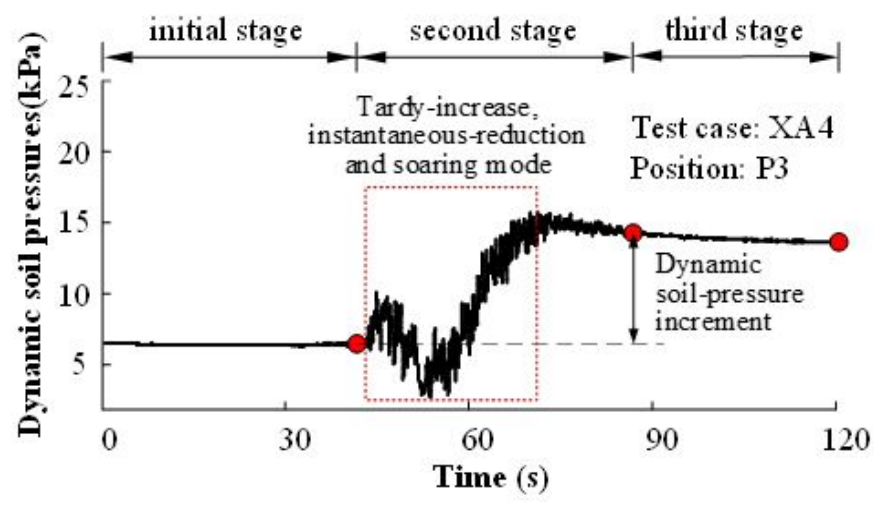

(c) Time-history curve during test case XA4

Figure 8: Time-histories of dynamic soil pressure at the top of sidewall.

The dynamic soil-pressure increments, as shown in Fig. 8, measured in different working conditions of tests are given in Table 7. It can be seen from the Table that the dynamic soil-pressure increments on the interface between loess and subway station have a certain regularity in the process of soil-structure interaction. First, the dynamic soil-pressure increments in side wall gradually increase with the PGA of input motions increase. Under input ground motions with different frequency spectrum, most of the dynamic soil-pressure increments induced by the Xi'an artificial waves are larger than those induced by Songpan and Taft ground motions because that the low frequency components of Xi'an artificial waves are more plentiful than other ground motions. Second, the dynamic soil-pressure increments on the top of side wall are significantly greater than those on other parts of side wall while the dynamic soil-pressure increments on the bottom of side wall are slightly less than those in the middle of side wall for most of test cases. It indicates that the soil-structure interaction on the top of structure is more intense during the shaking table tests. Third, the dynamic soil-pressure increments generated beneath the bottom plate (P4) are observably larger than those over the top plate (P5) due to the bottom plate bearing the gravity of the structure and the covering soil simultaneously. 
Table 7 Dynamic soil-pressure increments on the interface between loess and subway station

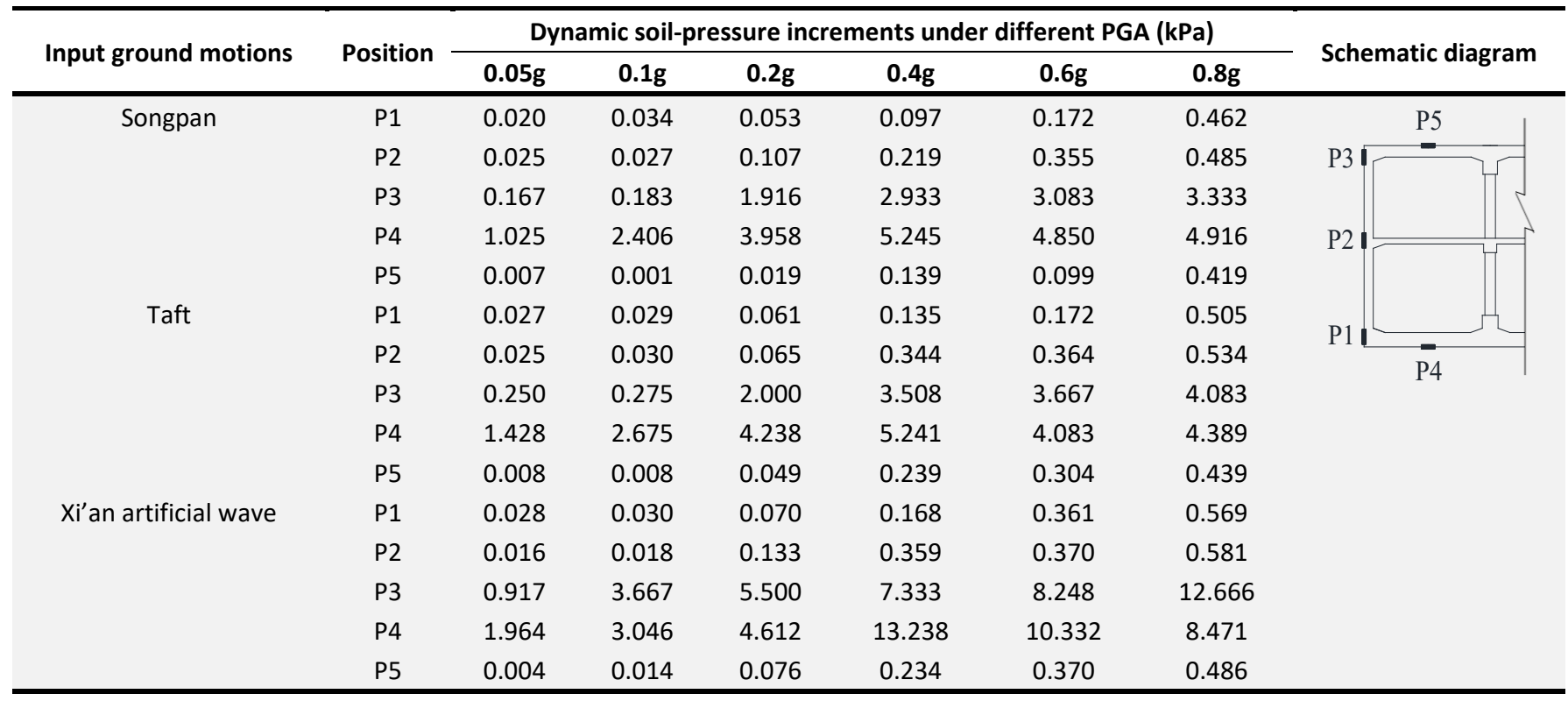

\subsection{Vertical settlement of the model system}

The settlements at different positions of the model system in the tests are collected by the laser sensors G1 and G2, which are placed at the surface of model soil, as shown in Fig. $3^{\odot}$. Table 8 shows the surface settlements of model soil in different working conditions. For most of test cases, the surface settlements of model soil increase with the PGA of input motions increase. Meanwhile, the surface settlements induced by the Xi'an artificial waves are larger than those induced by Songpan and Taft ground motions at the same PGA due to the plentiful low-frequency components in Xi'an artificial waves. It can also be seen from Table 8 that the surface settlements of model soil over the structure (G2) are always smaller than those away from the structure (G1) for all of the test cases, which means the model structure being lifted relatively during the shaking table tests.

Table 8 Settlements of model soil and uplift of model structure

\begin{tabular}{|c|c|c|c|c|c|c|c|c|c|}
\hline \multirow{2}{*}{$\begin{array}{l}\text { PGA of input } \\
\text { motions }\end{array}$} & \multicolumn{3}{|c|}{ Settlements of G1 (mm) } & \multicolumn{3}{|c|}{ Settlements of G2 $(\mathrm{mm})$} & \multicolumn{3}{|c|}{ Uplift of model structure $(\mathrm{mm})$} \\
\hline & Songpan & Taft & $\begin{array}{l}\text { Xi'an } \\
\text { wave }\end{array}$ & Songpan & Taft & $\begin{array}{l}\text { Xi'an } \\
\text { wave }\end{array}$ & Songpan & Taft & $\begin{array}{l}\text { Xi'an } \\
\text { wave }\end{array}$ \\
\hline $0.05 \mathrm{~g}$ & 0.002 & 0.009 & 0.001 & 0.001 & 0.005 & -0.003 & 0.001 & 0.004 & 0.004 \\
\hline $0.1 \mathrm{~g}$ & 0.005 & 0.077 & 0.130 & 0.003 & -0.008 & 0.075 & 0.002 & 0.085 & 0.055 \\
\hline $0.2 \mathrm{~g}$ & 0.165 & 0.627 & 1.059 & 0.065 & 0.044 & 0.968 & 0.099 & 0.583 & 0.091 \\
\hline $0.4 \mathrm{~g}$ & 1.219 & 4.144 & 11.020 & 0.760 & 2.158 & 6.770 & 0.458 & 1.986 & 4.250 \\
\hline $0.6 \mathrm{~g}$ & 1.322 & 1.426 & 12.823 & 1.000 & 1.155 & 12.047 & 0.322 & 0.271 & 0.776 \\
\hline $0.8 \mathrm{~g}$ & 1.983 & 4.790 & 11.086 & 1.756 & 4.405 & 10.860 & 0.228 & 0.385 & 0.225 \\
\hline
\end{tabular}

The uplift of model structure can be used to denote the difference values of the surface settlements between G1 and G2 in the tests (see Table 8). For the test cases with a lower PGA (e.g., PGA=0.05 g, $0.1 \mathrm{~g}$ or $0.2 \mathrm{~g}$ ), the uplift of model structure increase gradually as the PGA of input motions raise. However, the uplift of model structure have an obvious reduction for a higher PGA (e.g., $P G A=0.6 \mathrm{~g}$ or $0.8 \mathrm{~g}$ ). When $\mathrm{PGA}=0.4 \mathrm{~g}$, the uplift of model structure reached the maximum.

Fig. 9 shows the uplift of model structure as well as the difference of dynamic soil-pressure increments. It can be found that the difference values of dynamic soil-pressure increments between P4 (on the upper surface of top plate) and P5 (on the lower surface of bottom plate) have a similar variation law with the uplift of model structure. As the PGA of input motions raise, the difference values of dynamic soil-pressure increments increase when the PGA less-than $0.4 \mathrm{~g}$ while decrease when the PGA greater than $0.4 \mathrm{~g}$. It reaches the maximum when the PGA of input motions being equal to $0.4 \mathrm{~g}$. Hence, it is reasonably deduced that the ascending motions of model structure during the tests are powered by the difference values of dynamic soil-pressure increments between P4 and P5. 


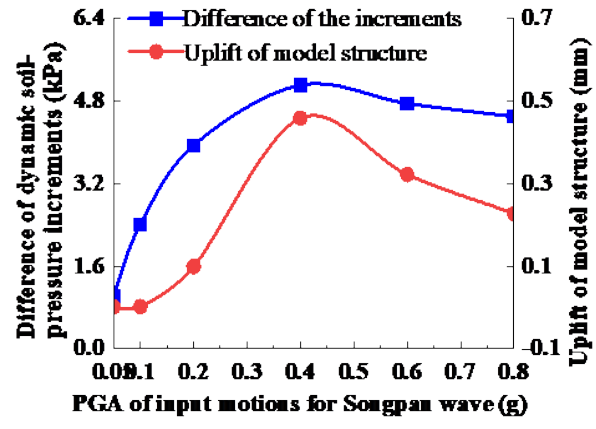

(a) Fitted curve under Songpan wave

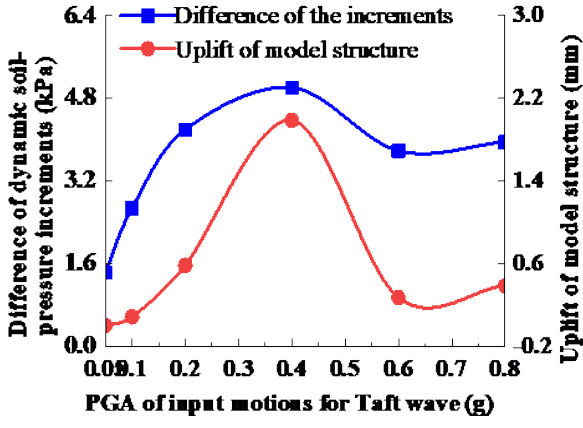

(b) Fitted curve under Taft wave

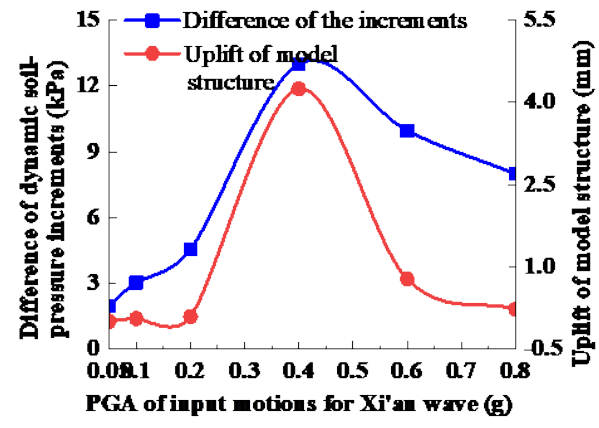

(c) Fitted curve under Xi'an wave

Figure 9 Difference of dynamic soil-pressure increments and uplift of model structure

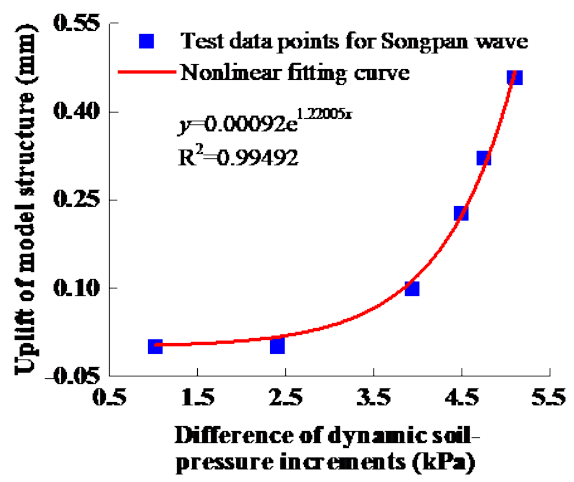

(a) Fitted curve under Songpan wave

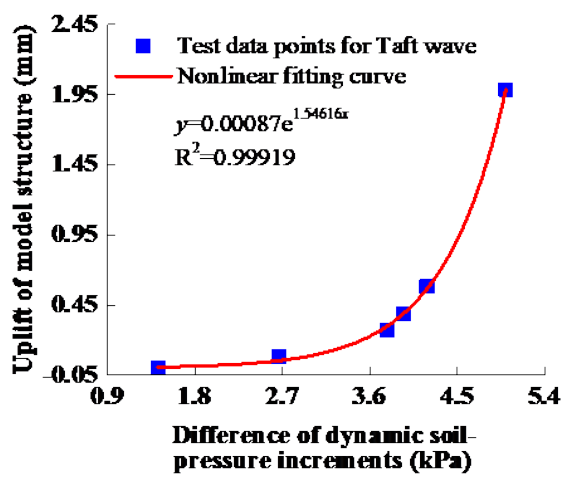

(b) Fitted curve under Taft wave

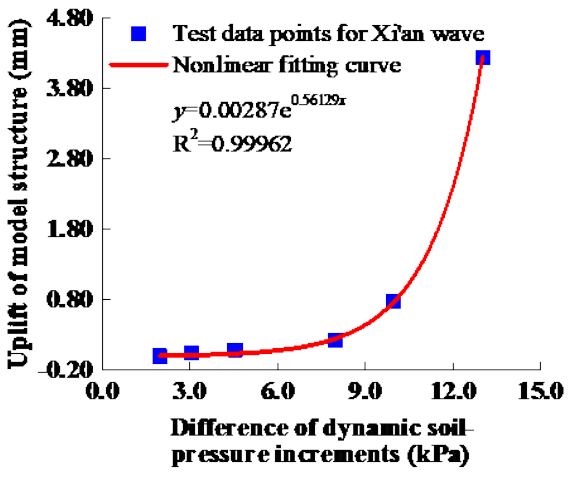

(c) Fitted curve under Xi'an wave

Figure 10 Relationship between the uplift of model structure and difference of dynamic soil-pressure increments

Furthermore, the nonlinear fitting method is used to quantitatively analyze the correlation between the uplift of model structure and the difference values of dynamic soil-pressure increments. The results of nonlinear fitting are shown in Fig. 10. It can be seen from the figure that an exponential relationship exists between the uplift of model structure and the difference values of dynamic soil-pressure increments.

\subsection{Macroscopic damage of loess and structure}

The crack development of ground surface and seismic damage of model structure are investigated in the shaking table tests and the results are shown in Fig. 11. After the completion of the test XA4 (Xi'an artificial wave with PGA=0.4 $\mathrm{g})$, the ground surface cracks right above the side walls of model structure begin to appear along longitudinal direction of the structure. After the completion of the test XA5 (Xi'an artificial wave with PGA=0.6 g), the ground surface cracks right above the end of model structure appears along transverse direction of the structure, meanwhile, the longitudinal cracks of ground surface develops more significantly. For the test XA6 (Xi'an artificial wave with PGA=0.8 g), the longitudinal and transverse cracks of ground surface right above the model structure are rapid expanding and connecting to each other to form a closed main crack. Around the main crack, several minor cracks appear and extend to far field away from the model structure, as shown in Fig. 11(a). After any of the tests, the width of the main crack is measured, as shown in Fig. 11(b). The largest width of measured main cracks is equal to $1.5 \mathrm{~cm}$, the difference value of vertical settlements between inside and outside of main crack reaches a maximum of $3.2 \mathrm{~cm}$.

Fig. $11^{\odot}$ shows the damage of the model structure after the shaking table tests. From the figure, the typical shear compression failure can be observed in the central column of the upper storey with obvious vertical cracks appearing. The concrete of the central column in the lower storey peels off a lot, and longitudinal stress reinforcement is exposed. The cracks in the intersections between the side wall and the top or bottom plate appear visibly and the steel bars embedded in the intersections are pulled out from the concrete. Junctions between central columns and the top or bottom plate are damaged most seriously. Excepting junctions between structural members, the rest parts of plates and side walls are undamaged. 


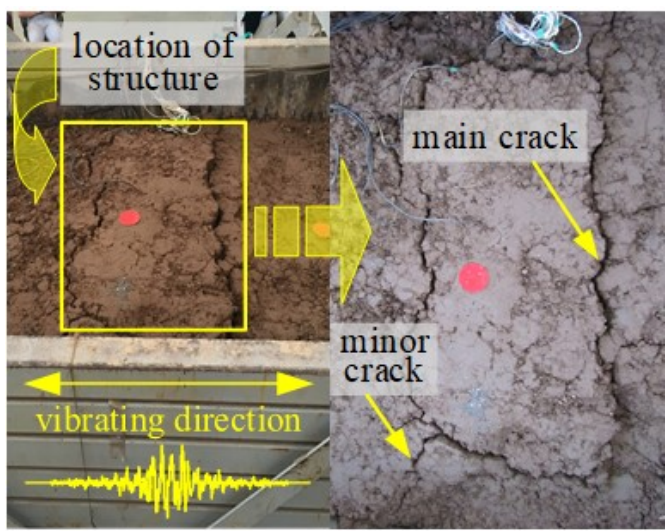

(a) Macroscopic damage of model site

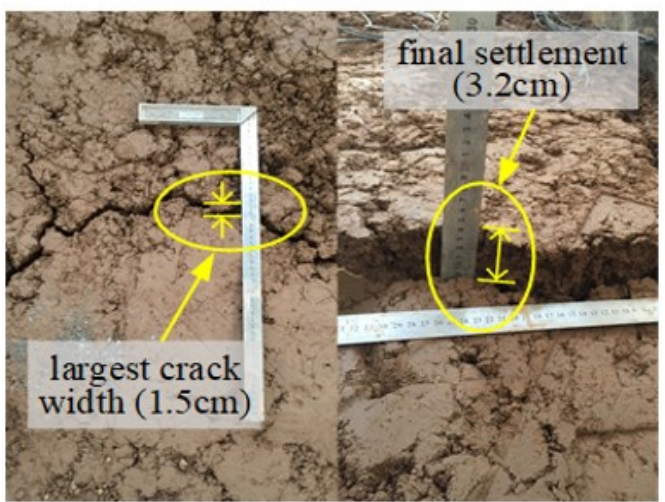

(b) Crack and settlement of ground

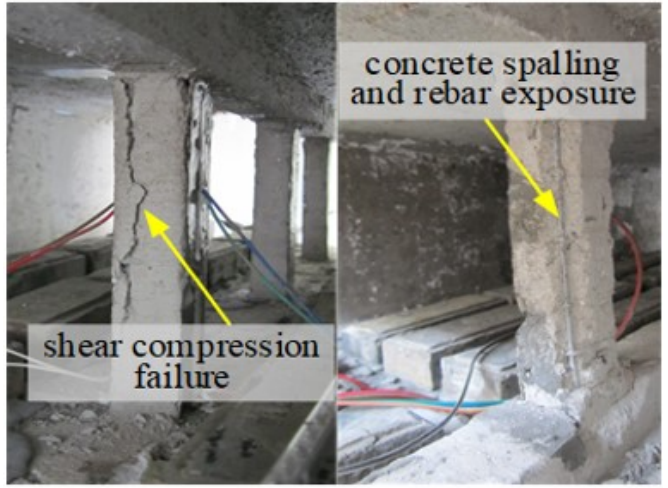

(c) Failure of model structure

Figure 11: Seismic damage of model site and structure.

\section{CONCLUSIONS}

In this paper, the seismic responses and damage characteristics of subway structure in loess ground are investigated through a series of shaking table tests on a large-scale structure model. From the test results, the following conclusions can be obtained:

1. The peak accelerations of model soil at the same depth increase observably with the increasing of the PGA of input motions. For the most of test cases, the peak acceleration increases gradually from the bottom to the top of model soil because of the amplification effect. The peak acceleration induced by the Xi'an artificial waves under a strong ground motion is greater than that induced by the Songpan and Taft ground motions due to the larger low frequency components in Xi'an artificial waves.

2. The gravity distribution of model structure has an important influence on its seismic response characteristics in the incongruous movement. So it is necessary to consider the influence of the gravity distribution on the seismic action of structural members during the seismic design of underground structure in loess area. 
3. From the overall perspective, the strain amplitudes in the top and bottom plate are smaller than those in side walls which are smaller than those in central columns because of the gravity and stiffness distribution of model structure. The strain amplitudes on the top of central columns are larger than those on the bottom of central column in the upper storey. On the contrary, in the lower storey, the result is reversed.

4. The dynamic soil-pressure increments in side wall gradually increase with the increasing of the PGA of input motions. Due to the soil-structure interaction on the top of structure being more intense during the shaking table tests, the dynamic soil-pressure increments on the top of side wall are greater than those on the other parts of side wall. The dynamic soil-pressure increments induced by the Xi'an artificial waves are generally larger than those induced by the Songpan and Taft ground motions because of more plentiful low-frequency components in the Xi'an artificial waves.

5. During the shaking table tests, the model structure is lifted relatively and the uplift of model structure have a similar variation law with the difference values of dynamic soil-pressure increments between P4 (over the top plate) and P5 (below the bottom plate). Further studies indicate that an exponential relationship exists between the uplift and the difference values.

6. After the shaking table tests, the typical shear compression failure and obvious vertical cracks occur in the central columns of the upper storey. Plenty of concrete is desquamated and many longitudinal stress reinforcements are exposed in the central columns of the lower storey. Junctions between the central columns and the top or bottom plate of model structure are damaged most seriously. Therefore, the effective seismic fortification measures should be adopted to insure the safety of the junctions during earthquake in loess area.

\section{Acknowledgements :}

This work was supported by the Natural Science Basic Research Plan in Shaanxi Province (No. 2020JM-234 and No. 2019JQ-689), the Science and Technology Plan of Xi'an Construction Committee (No. SZJJ2019-19) and the Fundamental Research Funds for the Central Universities, CHD (No. 300102280105).

The authors sincerely thank the staff at Jiangsu Province Key Laboratory of Civil Engineering and Disaster Prevention and Mitigation, attached to the Nanjing University of Technology.

Author's Contribuitions: Conceptualization, D Quan, S Chen and Y Wang; Methodology, D Quan, S Chai, S Chen and Y Wang; Investigation, D Quan and Y Wang; Writing original draft, D Quan and S Chai; Writing - review \& editing, S Chai and D Quan; Funding acquisition, D quan, S Chai, Y Wang; Resources, S Chen and Y Wang; Supervision, Y Wang.

Editor: Marcílio Alves.

\section{References}

Adalier, K.; Abdoun, T.; Dobry, R.; Phillips, R.; Yang, D.; Naesgaard, E. (2003). Centrifuge modeling for seismic retrofit design of an immersed tube tunnel. Journal of Physical Modelling on Geotechnics, 3(2), 23-32.

Baziar, M.H.; Moghadam, M.R.; Kim, D.S.; Choo, Y.W. (2014). Effect of underground tunnel on the ground surface acceleration. Tunnelling and Underground Space Technology, 44, 10-22.

Chen, G.X.; Zhuang, H.Y.; Du, X.L.; Liang, L.I.; Cheng, S. (2007). Analysis of large-scale shaking table test of dynamic soil-subway station interaction. Journal of Earthquake Engineering \& Engineering Vibration, 27(2), 171-176.

Chen, G.X.; Wang, Z.H.; Zuo, X.; Du, X.L.; Gao, H. (2013). Shaking table test on the seismic failure characteristics of a subway station structure on liquefiable ground. Earthquake Engineering \& Structural Dynamics, 42, 1489-1507.

Chen, G.X.; Chen, S.; Zuo, X.; Du, X.L.; Qi, C.Z.; Wang, Z.H. (2015). Shaking-table tests and numerical simulations on a subway structure in soft soil. Soil dynamic and earthquake engineering, 76, 13-28.

Chian, S.C.; Madabhushi, S.P.G. (2012). Effect of buried depth and diameter on uplift of underground structures in liquefied soils. Soil Dynamics and Earthquake Engineering, 41, 181-190. 
Cilingir, U.; Madabhushi, S.P.G. (2011). A model study on the effects of input motion on the seismic behaviour of tunnels. Soil Dynamics and Earthquake Engineering, 31(3), 452-462.

Dashti, S.; Hashash, Y.; Gillis, K.; Musgrove, M.; Walker, M. (2016). Development of dynamic centrifuge models of underground structures near tall buildings. Soil Dynamics and Earthquake Engineering, 86, 89-105.

Farahi, J.H.; Fardin, J.; Samadian, Z.M. (2018). Experimental study of burial depth effect on embedded pipe deformations in sandy slopes under dynamic landsliding. Soil Dynamics and Earthquake Engineering, 114, 281-297.

Hamayoon, K.; Keisuke, N.; Haruki, N.; Feng, Z. (2018). 1-g shaking table tests on seismic enhancement of existing box culvert with partial ground-improvement method and its 2D dynamic simulation. Soils and Foundations, 58(3), 563-581.

Han, C. (2011). Study on the response and design method of circular tunnel under severe earthquakes, Ph.D Thesis, Zhejiang University, Hangzhou.

Jafarzadeh, F.; Farahi Jahromi, H.; Abazari Torghabeh, E. (2010). Investigating Dynamic Response of a Buried Pipeline in Sandy Soil Layer by $1 G$ Shaking Table Test. International Journal of Civil Engineering, 8(2), 107-124.

Koseki, J.; Matsuo, O.; Koga, Y. (1997). Uplift behaviour of underground structures caused by liquefaction of surrounding soil during earthquake. Soils and Foundations, 37(1), 97-108.

Kang, G.; Tobita, T.; lai, S. (2014). Seismic simulation of liquefaction-induced uplift behavior of a hollow cylinder structure buried in shallow ground. Soil Dynamics and Earthquake Engineering, 64, 85-94.

Lanzano, G.; Bilotta, E.; Russo, G.; Silvestri, F.; Madabhushi, S.P.G. (2012). Centrifuge modeling of seismic loading on tunnels in sand. Geotechnical Testing Journal, 35, 1-16.

Li, D.Y.; Wang, B.M.; Lin, Y.C. (1996). Model test of structure, Science Press, Beijing.

Lin, G.; Zhu, T.; Lin, B. (2000). Similarity technique for dynamic structural model test. Journal of Dalian University of Technology, 40(1), 1-8.

Ling, D.S.; Guo, H.; Cai, W.J.; Han, C. (2012). Research on seismic damage of metro station with centrifuge shaking table model test. Journal of Zhejiang University (Engineering Science), 10, 1955-1961.

Liu, N.N.; Huang, Q.B.; Ma, Y.J.; Bulut, R.; Peng, J.B.; Fan, W.; Men, Y.M. (2017). Experimental study of a segmented metro tunnel in a ground fissure area. Soil Dynamics and Earthquake Engineering, 100, 410-416.

Liu, N.N.; Huang, Q.B.; Wang, L.; Fan, W.; Jiang, Z.K.; Peng, J.B. (2018). Dynamic characteristics research of a ground fissure site at Xi'an, China. Tunnelling and Underground Space Technology, 82, 182-190.

Ma, X.F.; Wang, G.B.; Wu, J.; Ji, Q.Q. (2017). Experimental Study on the Seismic Response of Subway Station in Soft Ground. Journal of Earthquake \& Tsunami, 11(1), 1750020.

Meymand, P.J. (1998). Shaking table scale model tests of nonlinear soil-pile-superstructure interaction in soft clay, Ph.D. Thesis, University of California, Berkeley.

Qin, L.K. (2010). Dynamic model of unsaturated loess and it's application to the seismic response of subway station, Ph.D Thesis, Chang'an University, Xi'an.

Quan, D.Z.; Wang, Y.H.; Jing, Y.L.; Yin, S.Z.; Ye, D. (2015). Numerical model and measuring position for subway stations in loess area. Technology for Earthquake Disaster Prevention, 10(1), 108-115.

Quan, D.Z.; Wang, Y.H.; Ma, P.B.; Jing, Y.L.; Chen, S. (2016). Spatial effect and frequency analysis on seismic response of subway station in loess ground. Journal of Vibration and Shock, 35(21), 102-112.

Rabeti Moghadam, M.; Baziar, M.H. (2016). Seismic ground motion amplification pattern induced by a subway tunnel: Shaking table testing and numerical simulation. Soil Dynamics and Earthquake Engineering, 83, 81-97.

Ritesh, K.; Kazuki, H.; Akihiro, T. (2019). Centrifuge testing to investigate effects of partial saturation on the response of shallow foundation in liquefiable ground under strong sequential ground motions. Soil Dynamics and Earthquake Engineering, 125 (No. 105728).

Sasaki, T.; Matsuo, O.; Kondo, K. (1999). Centrifuge model tests on uplift behavior of buried structures during earthquakes. Earthquake Geotechnical Engineering, 315-320. 
Tamari, Y.; Towhata, I. (2003). Seismic soil-structure interaction of cross sections of flexible underground structures subjected to soil liquefaction. Soils and foundations, 43(2), 69-87.

Tobita, T.; Kang, G.C.; lai, S. (2011). Centrifuge modeling of manhole uplift in a liquefied trench. Soil and Foundation, 51(6), 1091-1102.

Tsinidis, G.; Pitilakis, K.; Heron, C.; Madabhushi, G. (2013). Experimental and numerical investigation of the seismic behavior of rectangular tunnels in soft soils. $4^{\text {th }}$ ECCOMAS Thematic Conference on Computational methods in structural dynamics and earthquake engineering conference, Kos, Greece.

Wang, S.W.; Zhou, Y.M.; Mi, S.Y. (2013). Shaking Table Test of Muti-Story Subway Station Considering Soil-Structure Interaction. Advanced Materials Research, 694-697: 321-324.

Wang, Z.J.; Luo, Y.S.; Yang, Y.J. (2010). Study of dynamic structural characteristics of unsaturated loesses in different regions. Rock and Soil Mechanics, 31(8), 2459-2464.

Varghese, R.M.; Madhavi Latha, G. (2014). Shaking table tests to investigate the influence of various factors on the liquefaction resistance of sands. Natural Hazards, 73(3), 1337-1351.

Zhuang, H.Y.; Wang, X.; Miao, Y.; Yao, E.; Chen, S.; Ruan, B.; Chen, G.X. (2019). Seismic responses of a subway station and tunnel in a slightly inclined liquefiable ground through shaking table test. Soil Dynamics and Earthquake Engineering, 116, 371-385. 